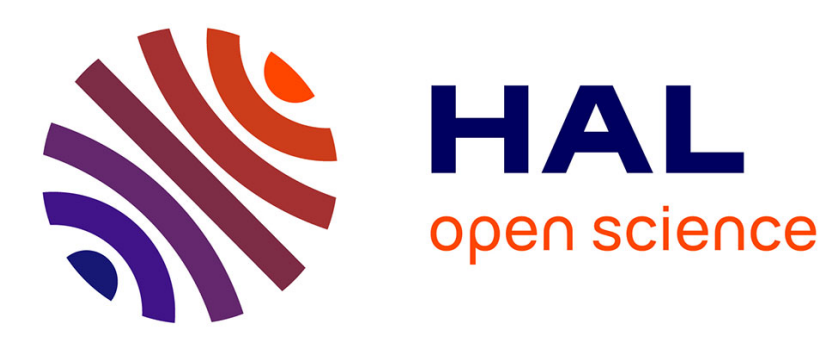

\title{
A Phase Correction for Long Distance Propagation Using Split-Step Methods in Non-Rectangular Domains
} Lucas Ligny, Zeina El Ahdab, Rémi Douvenot

\section{To cite this version:}

Lucas Ligny, Zeina El Ahdab, Rémi Douvenot. A Phase Correction for Long Distance Propagation Using Split-Step Methods in Non-Rectangular Domains. IEEE Antennas and Wireless Propagation Letters, 2021, 20 (12), pp.2476-2480. 10.1109/LAWP.2021.3115256 . hal-03354778

\author{
HAL Id: hal-03354778 \\ https://hal.science/hal-03354778
}

Submitted on 11 Oct 2021

HAL is a multi-disciplinary open access archive for the deposit and dissemination of scientific research documents, whether they are published or not. The documents may come from teaching and research institutions in France or abroad, or from public or private research centers.
L'archive ouverte pluridisciplinaire HAL, est destinée au dépôt et à la diffusion de documents scientifiques de niveau recherche, publiés ou non, émanant des établissements d'enseignement et de recherche français ou étrangers, des laboratoires publics ou privés. 


\title{
A Phase Correction for Long Distance Propagation Using Split-Step Methods in Non-Rectangular Domains
}

\author{
Lucas Ligny, Zeina El-Ahdab and Rémi Douvenot
}

\begin{abstract}
In this letter, an additional phase correction to Donohue and Kuttler's shift map theories is proposed. This correction enables a fast and accurate evaluation of the electromagnetic field at the receiver, both in terms of field amplitude and phase. Numerical tests are led in the troposphere, and the observed difference between the field computed with the corrected shift map and the field computed with a classic splitstep method is less than $-35 \mathrm{dBV} / \mathrm{m}$. The corrected shift map is also applied to a Radio Occultation configuration for which the phase information is important. The split-step wavelet method is applied in a reduced non-rectangular domain and shows good computational times.
\end{abstract}

Index Terms-Electromagnetic Propagation, Parabolic Wave Equation, Split-Step Methods, Radio Occultation.

\section{INTRODUCTION}

$\mathrm{T}$ HE analysis of the propagation of electromagnetic fields over long distances in inhomogeneous media is a difficult task. Indeed, some applications require a precise evaluation of the amplitude and the phase of the propagated field, such as the study of Radio Occultation (RO) [1]-[4].

Classic RO techniques are based on ray tracings between a GNSS satellite and a Low Earth Orbit (LEO) satellite and on the measurement of radio signals Doppler shifts induced by the atmosphere. Such techniques generally offer both fast and precise solutions [1]-[4]. However, these methods become unsuitable in the lower troposphere because of multipath phenomena [5]-[8].

Methods based on the resolution of the Parabolic Wave Equation (PWE) are also used to deal with such configurations [8]-[11]. These methods, called split-step or phase-screen, are workable even in the lower troposphere. Moreover, as electromagnetic fields fulfill the paraxial approximation in the context of $\mathrm{RO}$, they are considered as full-wave methods. Different techniques have been used to model boundaries while using these methods. The change of variables introduced by Beilis and Tappert [12] then used by Barrios [13] and by Donohue and Kuttler [14] allows us to take into account the curvature of Earth directly in the expression of the PWE. Another type of conformal mapping technique was introduced by Dozier [15] for acoustic applications and requires relatively small numerical range steps.

The authors are with the French Civil Aviation University (ENAC), Labo TELECOM-EMA, 7 avenue Edouard Belin, 31055, Toulouse, France (e-mail: ligny.lucas9@gmail.com; zeina.el-ahdab@enac.fr; remi.douvenot@enac.fr)
A more recent boundary modeling technique, which uses a coordinate system that follows the boundary, was introduced by Janaswamy [16] allowing bigger vertical steps.

This letter proposes an efficient split-step method to significantly gain in computational time and memory size while not losing any notable accuracy on the phase of the field. This is first achieved by reducing the propagation window around the primary ray (i.e. containing all the needed information), by using shift maps introduced by [14]. Since these shift maps only preserve the amplitude of the propagated field, a new correction term must be added to its phase at each propagation step of the method.

The structure of this paper is the following. In Section II, shift maps developed by Donohue and Kuttler [14] are reworked in order to develop a window-reducing, phasepreserving propagation method. In Section III, a validation of the method in the troposphere and an application to an $\mathrm{RO}$ configuration are proposed.

\section{Parabolic Wave Equation Transformation}

\section{A. Mathematical Model}

The geometry of the problem is shown in Fig. 1. In the Cartesian coordinate system $(x, y, z)$, the problem is assumed to be invariant to translations along $\hat{\boldsymbol{y}}$. The main direction of propagation is along $\hat{\boldsymbol{x}}$ and the vertical along $\hat{\boldsymbol{z}}$.

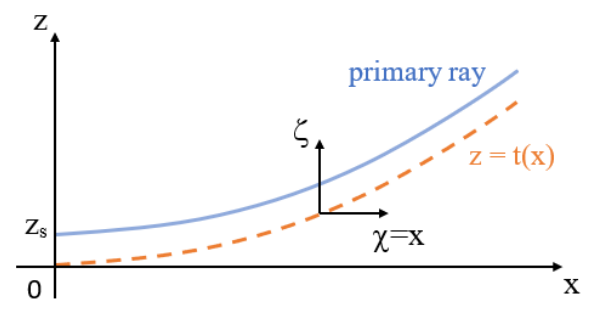

Fig. 1. The Cartesian coordinate system. The source is at $z=z_{s}$, and a receiver can be found following the primary ray (in blue). The dashed, orange line represents the change of variables described by equation (2).

The propagation medium is considered without sources and we assume an $e^{j \omega t}$ time dependency. The scalar Helmholtz equation in such a configuration gives [17]:

$$
\frac{\partial^{2} u}{\partial x^{2}}-2 j k_{0} \frac{\partial u}{\partial x}+\frac{\partial^{2} u}{\partial z^{2}}+k_{0}^{2}\left(n^{2}-1\right) u=0,
$$


where $k_{0}$ is the free-space wavenumber, $n(x, z)$ is the index of refraction and $u(x, z)$ represents a scalar component of the electric field. We want to work in a coordinate system that centers the propagation around the primary ray. The primary ray is defined as the emitted ray that takes the shortest path to the receiver.

The change of variables is expressed as follows [12]:

$$
\begin{gathered}
\chi=x \\
\zeta=z-t(x),
\end{gathered}
$$

with $t(x)$ the trajectory of the followed ray (see Fig. 1).

We denote respectively $\phi(\chi, \zeta) \equiv u(x, z), \nu(\chi, \zeta) \equiv$ $n(x, z)$ the field and the index of refraction expressed in the new coordinate system.

Split-step methods work only if there is no first derivative with respect to the vertical coordinate in the equation of propagation. One has to consider another field $\psi$ to meet such requirement, defined as follows:

$$
\psi(\chi, \zeta)=\phi(\chi, \zeta) e^{-j \theta(\chi, \zeta)},
$$

with $\theta(\chi, \zeta)$ a phase factor to be determined.

Applying (2) and (3) in (1), then keeping only forward propagating waves, we can write a new reduced form of the Helmholtz equation as:

$$
\begin{aligned}
& {\left[\frac{\partial}{\partial \chi}+j \frac{\partial \theta}{\partial \chi}-t^{\prime}\left(\frac{\partial}{\partial \zeta}+j \frac{\partial \theta}{\partial \zeta}\right)\right] \psi} \\
& =j k_{0}\left[1-\sqrt{\frac{1}{k_{0}^{2}}\left(\frac{\partial}{\partial \zeta}+j \frac{\partial \theta}{\partial \zeta}\right)^{2}+\nu^{2}}\right] \psi .
\end{aligned}
$$

Several approximations of the pseudo-differential operator, i.e. the square root term of equation (4), exist and lead to more or less robust expressions of the PWE. In Section II-B, Donohue and Kuttler's Wide-Angle Shift Map and Piecewise Linear Shift Map are studied.

\section{B. Phase Corrections for Shift Map Theories}

The continuity of the field $u(x, z)$ in the physical domain implies the continuity of $\phi(\chi, \zeta)$, which also implies the continuity of the $\psi(\chi, \zeta) e^{j \theta(\chi, \zeta)}$ entity.

Hence, the discontinuity in the transformed field $\psi(\chi, \zeta)$ should be compensated by the phase term $\theta(\chi, \zeta)$. In other words, in order to respect the continuity of the physical field (before transformation), a correction should be applied after each computation of the transformed field $\psi(\chi, \zeta)$ at each numerical step. Between two numerical steps, where $\chi_{1,2} \in\left[\chi_{1}, \chi_{2}\right]$, the correction to be applied to the field $\psi$ is reflected in the exponential term below:

$$
\psi_{2}\left(\chi_{1,2}, \zeta\right)=\psi_{1}\left(\chi_{1,2}, \zeta\right) e^{-j\left(\theta_{2}\left(\chi_{1,2}, \zeta\right)-\theta_{1}\left(\chi_{1,2}, \zeta\right)\right)} .
$$

We want to obtain an analytical expression of this correction term. In the following, the trajectory is supposed piecewise linear, which means that

$$
t^{\prime}(\chi)=T^{\prime} \text { and } t^{\prime \prime}(\chi)=0
$$

with $T^{\prime}$ a constant on each segment of the trajectory.
From Donohue and Kuttler's Wide-Angle Shift Map Theory, [14, eq. (13)], we have

$$
\theta(\chi, \zeta)=-k_{0} \zeta T^{\prime}+f(\chi)
$$

where [14, eq. (19)]

$$
f^{\prime}(\chi)=k_{0}\left(1-\frac{1}{2} T^{\prime 2}\right) .
$$

$T^{\prime}$ represents the local surface slope. It is related to the angle of incline $\beta$ as:

$$
T^{\prime}=\tan \beta .
$$

In order to integrate (8), one can remind the geometry of a changing surface slope (see Fig. 2).



Fig. 2. Geometry for propagating over a changing surface slope for discrete piecewise linear corner. $\beta_{1}$ and $\beta_{2}$ represent the local surface angles of incline. The local surface slopes are $T_{1}^{\prime}=\tan \beta_{1}$ and $T_{2}^{\prime}=\tan \beta_{2}$.

Since $f^{\prime}(\chi)$ is continuous on segments $S_{1}$ and $S_{2}$, its expression on each segment respectively is given by

$$
\begin{aligned}
& f_{S_{1}}^{\prime}(\chi)=k_{0}\left(1-\frac{1}{2} T_{1}^{\prime 2}\right) \\
& f_{S_{2}}^{\prime}(\chi)=k_{0}\left(1-\frac{1}{2} T_{2}^{\prime 2}\right) .
\end{aligned}
$$

Recall that the slope of the terrain is considered to be constant on each segment. Thus, $T_{1}^{\prime}$ and $T_{2}^{\prime}$ are constant and $f(x)$ can be deduced on each segment from (10a) and (10b) as follows:

$$
\begin{aligned}
& f_{S_{1}}(\chi)=k_{0}\left(1-\frac{1}{2} T_{1}^{\prime 2}\right) \chi \\
& f_{S_{2}}(\chi)=k_{0}\left(1-\frac{1}{2} T_{2}^{\prime 2}\right) \chi .
\end{aligned}
$$

Computing the discontinuity of $f(\chi)$ at $\chi=\chi_{1,2}$, from (11a) and (11b) we can write:

$$
f_{S_{2}}\left(\chi_{1,2}\right)-f_{S_{1}}\left(\chi_{1,2}\right)=k_{0}\left(\frac{1}{2} T_{1}^{\prime 2}-\frac{1}{2} T_{2}^{\prime 2}\right) \chi_{1,2} \text {. }
$$

Hence, the discontinuity of $\theta(\chi, \zeta)$ at $\chi=\chi_{1,2}$ can be expressed as:

$$
\begin{aligned}
& \theta_{2}\left(\chi_{1,2}, \zeta\right)-\theta_{1}\left(\chi_{1,2}, \zeta\right) \\
& \quad=k_{0} \zeta\left(T_{1}^{\prime}-T_{2}^{\prime}\right)+k_{0} \chi_{1,2}\left(\frac{1}{2} T_{1}^{\prime 2}-\frac{1}{2} T_{2}^{\prime 2}\right)
\end{aligned}
$$

Finally, replacing (13) in (5), the expression of the phase correction to apply to the field $\psi(\chi, \zeta)$ at each numerical step is:

$$
\begin{aligned}
& \psi_{2}\left(\chi_{1,2}, \zeta\right)=\psi_{1}\left(\chi_{1,2}, \zeta\right) \\
& \quad \times e^{-j k_{0} \zeta\left(T_{1}^{\prime}-T_{2}^{\prime}\right)} e^{-j k_{0} \chi_{1,2}\left(\frac{1}{2} T_{1}^{\prime 2}-\frac{1}{2} T_{2}^{\prime 2}\right)}
\end{aligned}
$$


It can be seen that the second right-hand term in (13) is missing in the phase correction proposed by Donohue and Kuttler [14, eq. (27)]. They have only considered the discontinuity in the $\zeta$ dependent component of $\theta(\chi, \zeta)$, but have neglected all discontinuities in the $\chi$ dependent term.

This correction can also be added to Donohue and Kuttler's Piecewise Linear Shift Map Theory. Considering,

$$
\begin{gathered}
\theta(\chi, \zeta)=-k_{0}^{\bmod } \zeta T^{\prime}+f(\chi) \\
f^{\prime}(\chi)=k_{0}^{\bmod }\left(1-T^{\prime 2}\right) \\
k_{0}^{\text {mod }}=\frac{k_{0}}{\sqrt{1+T^{\prime 2}}}
\end{gathered}
$$

the phase correction can now be written:

$$
\begin{aligned}
& \psi_{2}\left(\chi_{1,2}, \zeta\right)=\psi_{1}\left(\chi_{1,2}, \zeta\right) \\
& \quad \times e^{-j k_{0} \zeta\left(\sin \beta_{1}-\sin \beta_{2}\right)} e^{-j k_{0} \chi_{1,2}\left(\frac{1}{2} T_{1}^{\prime 2}-\frac{1}{2} T_{2}^{\prime 2}\right)} .
\end{aligned}
$$

Note that the last term in (16) is identical to the one in (14). The phase correction aims to force phase continuity in a space that follows $t(x)$, here the primary ray. It is natural to take into account $k_{0}$ and not the modified wavenumber $k_{0}^{\text {mod }}$, because $k_{0}^{\text {mod }}$ already takes into account the change of variables [14]. So considering it in the phase correction in $\chi$ would erroneously amount to counting this change of variables twice.

In order to check the consistency of the above formulations, a validation is first proposed in Section III-A, and an application to an RO configuration in Section III-B.

\section{NUMERICAL RESUlTS}

\section{A. Validation in the Troposphere}

In this section, validation of the phase correction obtained in Section II is presented. Please note that for all of the simulations in this section, results are excellent in terms of field amplitudes, and bring additional validation to Donohue and Kuttler's original theory. Indeed, we observe a maximum of $-50 \mathrm{dBV} / \mathrm{m}$ amplitude difference between fields calculated with and without shift maps. In this letter, these results are not displayed because we want to evaluate the mathematical difference between the two fields, taking into account both amplitude and phase of the electromagnetic field. As we wish to provide a validation of our equations, computation times and memory space are not presented in this section. Please check the next section to find such results.

Simulations are performed over $50 \mathrm{~km}$ for a maximum altitude of $512 \mathrm{~m}$. The source is a complex source point characterized by an altitude $z_{s}=250 \mathrm{~m}$, a belt $W_{0}=3 \mathrm{~m}$ $(\approx 15 \lambda)$ and a position according to the main direction of propagation $x_{s}=-500 \mathrm{~m}$. The horizontal and vertical steps are fixed at $\Delta x=200 \mathrm{~m}$ and $\Delta z=0.2 \mathrm{~m}$. Simulations run with an horizontal polarization, an apodization factor equal to 1 and disregard the influence of the ground, which is therefore removed. The atmosphere is represented by a linear refractivity, with parameters $N_{0}=330 \mathrm{~N}$-units the modified index of refraction on the ground and $C_{0}=0.05 \mathrm{~N}$-units $/ \mathrm{m}$ the index gradient. Note that $C_{0}$ has the magnitude of the index gradient of a standard atmosphere (in absolute value).
The signal frequency is $f=1.575 \mathrm{GHz}$. The trajectory of the primary ray is defined by [18]

$$
t(x)=z_{s}+\frac{C_{0} 10^{-6}}{2} x^{2} .
$$

The computation window of the field, corresponding to the band around the primary ray containing the desired section of the field, is considered maximum. Results for propagation using Donohue and Kuttler's shift maps are presented in Fig. 3 and Fig. 4.

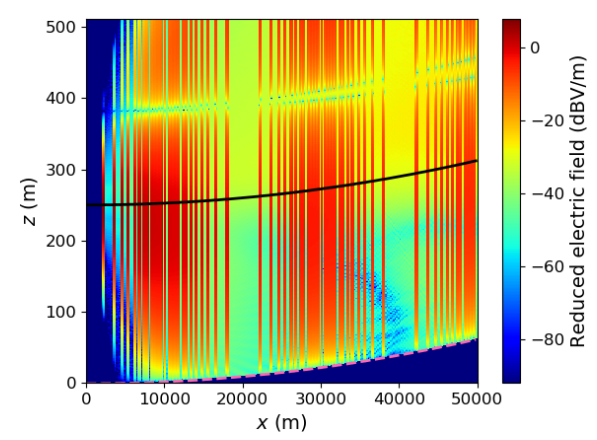

Fig. 3. Difference between the reduced field $u$ computed with the WideAngle Shift Map and the reduced field computed with a classic split-step method. The black line corresponds to the primary ray, and the dashed, pink line represents the limits of the computation window.

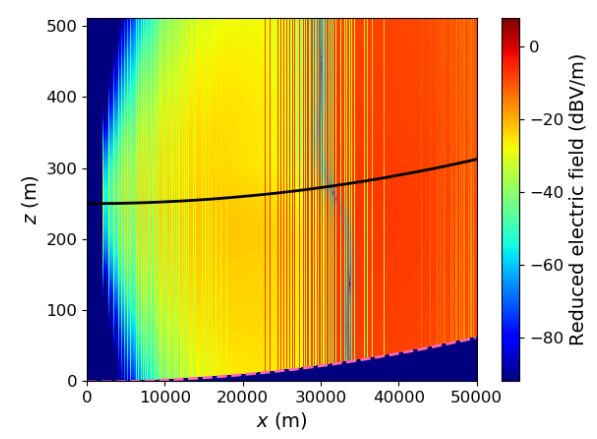

Fig. 4. Difference between the reduced field $u$ computed with the Piecewise Linear Shift Map and the reduced field computed with a classic split-step method.

In Fig. 3 (resp. Fig. 4) is represented the difference between the field computed with the Wide-Angle (resp. Piecewise Linear) Shift Map and the field computed with a classic split-step method, expressed in $\mathrm{dBV} / \mathrm{m}$, in the initial coordinate system $(x, z)$ and within the computation window.

We observe strong phase errors in both figures. In Fig. 3 these errors resemble periodic phase jumps, while in Fig. 4 they seem to accumulate starting from the source. In both cases, the phase information is lost at the receiver.

In Fig. 5 is represented the difference between the field computed with the Piecewise Linear Shift Map with the phase correction of Section II, and the field computed with a classic split-step method, expressed in $\mathrm{dBV} / \mathrm{m}$, in the initial coordinate system $(x, z)$ and within the computation window.

Results are good. The maximum error (difference) is -35 $\mathrm{dBV} / \mathrm{m}$, which is continuous and cumulative from the source. 


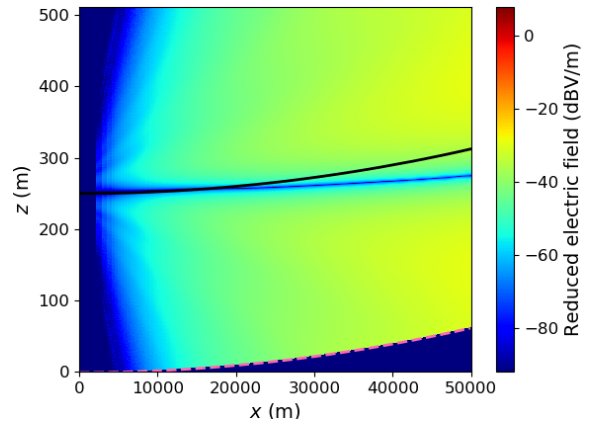

Fig. 5. Difference between the reduced field $u$ computed with the Piecewise Linear Shift Map plus the phase correction of Section II and the reduced field computed with a classic split-step method.

The root mean square error (RMSE) on the last vertical is $-25 \mathrm{~dB}$. These errors attenuate with larger step sizes and larger apodization windows.

Since the phase correction brought to Donohue and Kuttler's shift maps are numerically validated, the study of an $\mathrm{RO}$ configuration is proposed in Section III-B to provide a useful application of the method.

\section{B. Radio Occultation}

RO simulations are run on a computer equipped with an i5$8250 \mathrm{U}$ processor and a RAM of $8 \mathrm{~GB}$. The total simulation is performed over a distance of $4,260 \mathrm{~km}$, for a vertical extent of $24 \mathrm{~km}$ on either side of Earth's ground. The altitude of the GNSS satellite is $z_{\mathrm{GNSS}}=15 \mathrm{~km}$ and that of the LEO is $z_{\mathrm{LEO}}=0 \mathrm{~km}$. The horizontal and vertical steps are fixed at $\Delta x=1000 \mathrm{~m}$ and $\Delta z=2 \mathrm{~m}$. The two satellites communicate on the frequency $f=1.57542 \mathrm{GHz}$. Earth's atmosphere is represented by the ITU P835-6 profile, which is the reference atmosphere for gas attenuation [19]. Earth is considered to be a PEC and its relief is not taken into account. The apodization factor is fixed at 1 .

In Fig. 6 is represented the electric field, expressed in $\mathrm{dBV} / \mathrm{m}$, computed with a split-step wavelet method [20] in the initial coordinate system $(x, z)$. Numerical errors appear on both sides of the computation band (with no impact on the propagation). The refraction of the rays and the occultation of the field due to Earth are particularly visible.

In Fig. 7 is represented the electric field, expressed in $\mathrm{dBV} / \mathrm{m}$, computed with the Piecewise Linear Shift Map that takes into account the phase correction of Section II (and using the same split-step wavelet propagator as in Fig. 6 ) in the initial coordinate system $(x, z)$ and within the computation window. Note that reducing the computation window does not affect the results of the field obtained. The RMSE on the last vertical is $-20 \mathrm{~dB}$. The gain in computation time between the two simulations is presented in Table I.

TABLE I

Comparison on Computation Time And Memory Space

\begin{tabular}{|c|c|c|}
\hline Propagation $\left(E_{y}\right)$ & Computation Time & Memory Space \\
\hline Total Window & $1 \mathrm{~h} 43 \mathrm{~min}$ & $3,996 \mathrm{Mo}$ \\
Reduced Window & $24 \mathrm{~min}$ & $833 \mathrm{Mo}$ \\
\hline
\end{tabular}

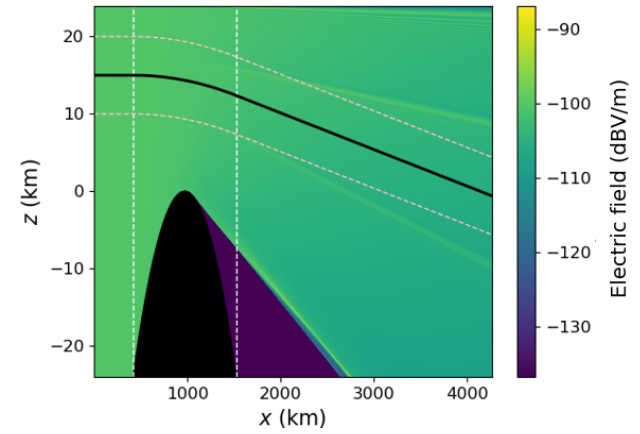

Fig. 6. Electric field $E_{y}$ computed with a classic split-step method in an RO configuration. The vertical, dashed, white lines represent the domain of propagation above Earth (surface filled with black).

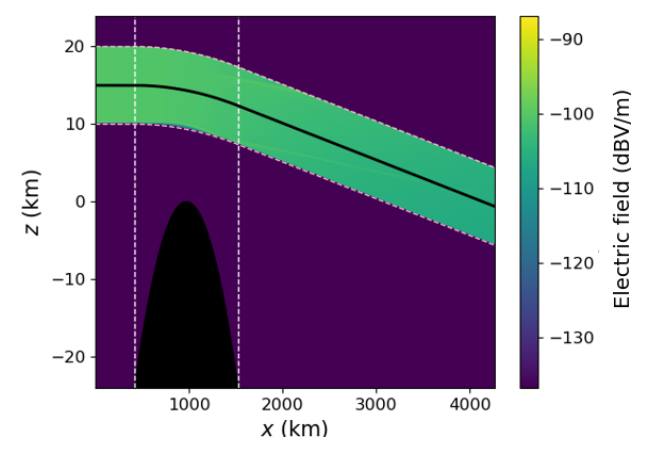

Fig. 7. Electric field $E_{y}$ computed with the Piecewise Linear Shift Map plus the phase correction of Section II in an RO configuration.

\section{CONCLUSION}

This letter presents a fast and accurate method that was developed to perform a full-wave propagation over long distances. Inspired by the work of Donohue and Kuttler, we have extracted the exact phase correction term to apply within the split-step method and we have managed to recover the phase information at the receiver. This has allowed us to reduce the propagation window around the primary ray and thus to reduce computation costs, which are usually high when studying an RO configuration. The next steps to apply this technique to RO is to develop fast techniques to extract Doppler and group delay from the calculated field.

\section{REFERENCES}

[1] E. R. Kursinski, G. A. Hajj, K. R. Hardy, J. T. Schofield, and R. P. Linfield, "Observing Earth's atmosphere with radio occultation measurements using the global positioning system," J. Geophys. Res., Atmos., vol. 102, pp. 23429-23465, Oct. 1997.

[2] C. Rocken, R. Anthes, M. Exner, D. Hunt, S. Sokolovskiy, R. Ware, M. Gorbunov, W. Schreiner, D. Feng, B. Herman, Y. H. Kuo, and $\mathrm{X}$. Zou, "Analysis and validation of GPS/MET data in the neutral atmosphere," J. Geophys. Res., Atmos., vol. 102, pp. 29849-29866, Dec. 1997.

[3] S. V. Sokolovskiy, "Inversions of radio occultation amplitude data," Radio Sci., vol. 35, no. 1, pp. 97-106, 2000.

[4] R. Kursinski, G. Hajj, S. Leroy, and B. M. Herman, "The GPS radio occultation technique," Terr. Atmos. Ocean. Sci., vol. 11, p. 53, Jan. 2001.

[5] M. E. Gorbunov, S. V. Sokolovskiy, and L. Bengtson, "Advanced algorithms of inversion of GPS/MET satellite data and their application to reconstruction of temperature and humidity," Tech. Rep. 211, Max Planck Inst. for Meteorol., Hamburg, Germany, 1996. 
[6] M. E. Gorbunov and A. S. Gurvich, "Microlab-1 experiment: multipath effects in the lower troposphere," J. Geophys. Res, vol. 103, no. D12, pp. 13,819-13,826, 1998.

[7] M. E. Gorbunov, A. S. Gurvich, and L. Kornblueh, "Comparative analysis of radioholographic methods of processing radio occultation data," Radio Sci., vol. 35, no. 4, pp. 1025-1034, 2000

[8] S. V. Sokolovskiy, "Modeling and inverting radio occultation signals in the moist troposphere," Radio Sci., vol. 36, no. 3, pp. 441-458, 2001.

[9] A. S. Jensen, M. S. Lohmann, H.-H. Benzon, and A. S. Nielsen, "Full spectrum inversion of radio occultation signals," Radio Sci., vol. 38, no. 3, 2003.

[10] M. S. Lohmann, A. S. Jensen, H.-H. Benzon, and A. S. Nielsen, "Application of window functions for full spectrum inversion of crosslink radio occultation data," Radio Sci., vol. 41, 2006.

[11] M. E. Gorbunov, O. A. Koval, and A. E. Mamontov, "Method of spherical phase screens for modeling the propagation of diverging beams in inhomogeneous media," Izvestiya, Atmospheric and Oceanic Physics, vol. 56, no. 1, pp. 52-60, 2020

[12] A. Beilis and F. D. Tappert, "Coupled mode analysis of multiple rough surface scattering," J. Acoust. Soc. Amer, vol. 66, pp. 811-826, 1979.

[13] A. E. Barrios, "A terrain parabolic equation model for propagation in the troposphere," IEEE Trans. Antennas Propag., vol. 42, no. 1, pp. 90-98, 1994

[14] D. Donohue and J. R. Kuttler, "Propagation modeling over terrain using the parabolic wave equation," IEEE Trans. Antennas Propag., vol. 48, no. 2, pp. 260-277, 2000 .

[15] B. L. Dozier, "PERUSE: A numerical treatment of rough surface scattering for the parabolic wave equation," J. Acoust. Soc. Amer. vol. 75 , no. 5 , pp. 1415-1432, 1982.

[16] R. Janaswamy, "A curvilinear coordinate-based split-step parabolic equation method for propagation predictions over terrain," IEEE Trans. Antennas Propag., vol. 46, no. 7, pp. 1089-1097, 1998.

[17] F. D. Tappert, "The parabolic approximation method," Wave Propaga tion and Underwater Acoustics, vol. 70, pp. 224-287, 1977.

[18] L. Boithias, Radiowave propagation. McGraw-Hill, 1987.

[19] ITU, "Reference standard atmospheres," tech. rep., ITU-R P.835-6, 2017.

[20] H. Zhou, R. Douvenot, and A. Chabory, "Modeling the long-range wave propagation by a split-step wavelet method," J. Comput. Phys., vol. $402,2020$. 